\section{The interpersonal effects of emotion on rejection of severely unfair ultimatum proposal}

\author{
Davide Pietroni, Sibylla Hughes Verdi, Felice Giuliani, Angelo Rosa, \\ Fabio Del Missier and Riccardo Palumbo \\ (Author affiliations can be found at the end of the article)
}

\section{Severely unfair ultimatum proposal}

Received 30 March 2021 Revised 18 June 2021 Accepted 9 July 2021

\begin{abstract}
Purpose - The purpose of this study is to investigate how the emotion expressed by a fictitious proposer influences the responder's decision to accept or reject a severely unfair deal, represented by the splitting of a predetermined sum of money between the two players during an ultimatum game (UG). Rejection leads both parts to dissipate that sum. Critically the authors consider the situation in which both players have the best alternative to negotiation agreement (BATNA), which simulates a backup plan to rely on in case of no agreement.

Design/methodology/approach - The participants played a UG and, to foster the ecological validity of the paradigm, the parts could both rely on a more or less generous BATNA. The critical manipulation was the emotion expressed by the proposer while their BATNA was either hidden (Exp. 1) or communicated (Exp. 2).

Findings - The proposer's emotions influenced participants' own emotions, affected their social evaluations about the proposer, the desire for future interactions with the proposer and were used to infer the proposer's BATNA when it was unknown. In this latter case, proposers' emotions and in particular his/her happiness, decreased dramatically the participants' tendency to reject even severely unfair offers.

Originality/value - Past research on UG has been predominantly aimed to investigate the effect of responders' emotions or the effects of responders' emotions on the proposer, devoting little attention to how the critical responder's acceptance/rejection decision might be affected by the proposer's emotion. Especially in the ecological situation where the parts have a BATNA in case of non-agreement.
\end{abstract}

Keywords Happiness, Anger, Rejection, Ultimatum game, BATNA, Interpersonal effects of emotion

Paper type Research paper

\section{Introduction}

The ultimatum game (UG) models the crucial last stage of a negotiation, where the final offer can be accepted or rejected (Arvanitis et al., 2019). The decision to reject positive offers on the UG has been attributed to emotional reactions to unfair treatment that overrides purely economic considerations (Koenings and Tranel, 2007; Yamagishi et al., 2009; Reed et al., 2020). Literature consistently shows that unfair offers induce anger and their rejection relates to aggression and conflict escalation, to such an extent that UG has been proposed as an anger-induction paradigm (Gilam et al., 2019). Therefore, recent research effort has been dedicated to investigating factors associated with a greater propensity to accept even unfair offers (Paz et al., 2017; Park, 2021; Ng et al., 2019; Arvanitis et al., 2019).

(C) Davide Pietroni, Sibylla Hughes Verdi, Felice Giuliani, Angelo Rosa, Fabio Del Missier and Riccardo Palumbo. Published by Emerald Publishing Limited. This article is published under the Creative Commons Attribution (CC BY 4.0) licence. Anyone may reproduce, distribute, translate and create derivative works of this article (for both commercial and non-commercial purposes), subject to full attribution to the original publication and authors. The full terms of this licence may be seen at http://creativecommons.org/licences/by/4.0/legalcode
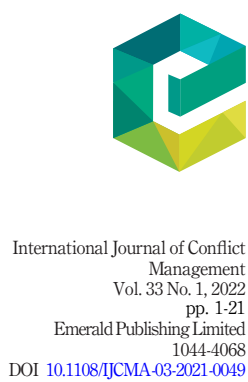
IJCMA 33,1

Dunn et al. (2012) found that the perception and regulation of bodily-based emotional biasing signals ("gut feelings") partly shape financial decision-making on the UG. Measuring the players' skin conductance responses as an autonomic index of affective state, they found that skin conductance activity was higher for unfair offers and that this was associated with a rejection of unfair offers. This lends support to the notion that rejection is related to an emotional response such as frustration and this could have strong implications for conflict studies, as conflict escalation often just starts with rejection, i.e. after the failure of some sort of ultimatum coordination trial.

Until about a dozen years ago, effects of emotion on the rejection rates of ultimatum proposals have been studied, as by Dunn et al. (2012), almost exclusively through an "intrapersonal" perspective, i.e. investigating the effect of the responders' own affective state on his/her decision to reject or accept the offer (for a review see Reed et al., 2020). In synthesis, this research found that sadness resulted in higher rejection rates of unfair offers while happiness was not associated with any systematic bias in decision-making (Harlé and Sanfey, 2007).

The current study is instead based on the seminal studies of Van Kleef $e t$ al. (2004a), which successfully adopted also an "interpersonal" perspective to study the effect of emotion in negotiation and economic games, focusing on the effect of the counterparts' emotion on the agent of a strategic decision. Consistent with the social functional approach, this line of research shows, with some important exceptions and distinctions, that decisionmakers facing an angry opponent make more cooperative strategic decisions (e.g. formulating higher offers in a negotiation) than those with a non-emotional counterpart, whereas decision-makers interacting with a happy counterpart make more competitive decisions (e.g. make lower concessions).

Unfortunately, most research on the interpersonal effects of emotions in the UG has focused on the effects of the respondent's emotions on the proposer's allocation decision, neglecting the effects of the proposer's emotions on the responder and not considering the source-specific effects of these emotions on the UG crucial final decision. The present research aspires to contribute to filling this gap.

\section{Literature review and hypotheses development}

\subsection{The role of interpersonal emotion of the proposer's}

Van Dijk et al. (2008), investigated the interpersonal effect of responders' anger and happiness on the proposers' offer on the UG. Their results corroborate the notion that when the responder expresses anger, the proposer formulates better offers to reduce the rejection risk, as anger could be interpreted as a signal that the responder has a high limit which could drive her or him to be tough and demanding. The authors also found evidence for emotional contagion, meaning that the proposer became angrier when confronted with an angry responder and happier with a happy one. This is the reason why the authors found that expressing anger may backfire in the long run.

Other studies have investigated the effect of interpersonal emotions on the proposer from different perspectives. Lelieveld et al. (2012) focused on anger and disappointment expressed by the responder as a consequence of the proposer's offer, whereas Nelissen et al. (2011) have shown that the proposer's allocation decision can be influenced by anticipated guilt or fear, depending, respectively, on the concern of being unfair or being rejected.

Despite these strides forward, the literature is still mostly neglecting the investigation of the interpersonal effect of the proposer's expressed emotion on the responder's decision in a UG. The general lack of interest in this aspect seemed to us a void that merits further investigation because studying the interpersonal effect of emotion on the responder means 
studying the effect of emotion on the protagonist of the UG. In fact, the responder is the one who must make the ultimate decision that could potentially instigate conflict through the rejection, depending on how fair the offer is perceived (Zheng et al., 2017 for a review on this topic). This research gap has been also acknowledged by Handgraaf et al. (2003), who have highlighted that the lack of focus on the responder is a missed opportunity to understand the interpersonal behavior in bargaining contexts. Since then, only little advancements have been made to fill this gap.

To the best of our knowledge, only three studies have specifically investigated how interpersonal primary emotions expressed by the proposer affect the responder's acceptance rate. Mussel et al. (2013), manipulating agents' facial expressions, have found that offers from proposers expressing anger have less likelihood to be accepted compared to neutral and smiling expressions. Besides the effect of the offer fairness, the whole range of possible offers, from the most generous ( 7 cents/3 cents) to the most unfair ( 1 cent/ 9 cents), was affected by this emotional effect. The hypothesis was that a smiling counterpart is perceived as friendly and bearer of positive intentions, whereas an angry counterpart is perceived as unfriendly and bearer of negative intentions. On the same stream of research, Liu et al. (2016) have found that proposer's sadness, disgust and fear also tend to reduce the responder's acceptance rate. However, in this case, the effect was explained in terms of emotional priming. Specifically, positive primed emotions may lead the responder to focus on the positive aspects of the offer such as the monetary reward, attenuating the focus on negative aspects such as the potential unfairness. On the contrary, negative primed emotions may lead to the opposite pattern.

Another study (Mussel et al., 2014) has investigated the effect of smiling and non-smiling proposers' expressions measuring responders' brain activity through electroencephalography, a technique that allows measuring the activity of the brain cortex. The study focused on a brain signal called feedback-related negativity which is indicative of an event that is "worse than expected." In fact, they have found that the amplitude of the signal increased as the offer became more unfair, indicating a greater violation of their expectation. Interestingly, smiling faces tend to reduce the amplitude of this signal, indicating a modulation of the negative feelings triggered by the violation of the expectation. At a behavioral level, Mussel et al. (2014) have also found that when the proposal comes from a smiling face, in some cases, it has a higher probability to be accepted, although the effect is rather small. The proposed explanation is that a positive emotion attenuates the negative feelings triggered by the unfairness of the offer.

From a social functional perspective of emotional expressions (Van Kleef, 2010; Van Kleef and Côté, 2018), it could seem at best a mere experimental exercise to investigate the interpersonal effect of emotion on the responder, as the information he/she can infer from the proposer's emotional expressions cannot be used strategically to modulate accordingly his/her counterproposal as he/she can only make the final decision to reject or accept the other's last proposal. However, such an argument seems to underestimate the "irrationality" of human decision-making (and the human drive to punish individuals that are perceived as unfair; Burnham, 2007), and therefore it could lead to the same conceptualization errors which undermined the predictive strength of the normative economic theory, which claims that every positive offer should not be rejected despite any fairness considerations. Instead, fairness assessment has been widely demonstrated to be critical (Bahrya and Wilson, 2006; Solnick and Schweitzer, 1999; Van Dijk et al., 2004) and, in line with other studies, we hypothesize that it could also significantly be affected by the emotional expression of the proposer.

\section{Severely unfair ultimatum proposal}


IJCMA

33,1

Relying on the results and interpretations previously reported on the effects of interpersonal primary emotions expressed by the proposer on the responder's acceptance rate (Liu et al., 2016; Mussel et al., 2013, 2014) and triggering the participants' tendency for rejection by proposing a severely unfair offer ( 85 chips for the proposer and 15 chips for the participant), we state our first hypothesis as follows:

H1. The proposers' anger expression would negatively influence the responders' affective state and impression about the other increasing the rejection rate compared to the control group.

On the other hand, given the marked unfairness of the ultimate offer, we state our second hypothesis as follows:

H2. The proposers' happiness expression would positively affect the responders' emotions and impressions but not enough to significantly reduce the rejection rate compared to the control group.

\subsection{Best alternative to the negotiation agreement, emotions and ultimatum game}

With the purpose of increasing the predictive strength of laboratory studies, some research on the interpersonal effects of emotion in negotiation tasks has tried to emulate more closely real-life situations by incorporating a "consolation prize" or a plan $\mathrm{B}$, called the best alternative to the negotiation agreement (BATNA) which represents a payoff of the variable entity that the parts can obtain in case of non-agreement (Van Dijk et al., 2008; Pietroni et al., 2008). The negotiation behavior investigated in an economic and social vacuum, where the only payoffs obtainable could be generated exclusively through the focal interaction, seem to oversimplify the paradigm limiting its ecological validity (Knez and Camerer, 1995). Moreover, to the best of our knowledge, the interpersonal effect of emotion on the responder has never been investigated in UGs including agents' BATNA.

As one of the most common forms of advice to an agent preparing for a negotiation is never enter the arena before having well defined your own BATNA (Fisher et al., 2011), it follows that the research in this area should not neglect to provide an explicit BATNA to the participants. According to this perspective, research on the interpersonal effects of emotion proposed that, among the set of different sources which can elicit the expresser's emotion (like, for example, the proposal, the proposer and the situation), one should also include the expresser's own BATNA, i.e. the emotions the expresser shows to the counterpart while taking into consideration his/her plan B available in case of failure of the interaction with the counterpart (Pietroni et al., 2009). Because of this important inclusion, the signaling function of the emotion may also help the parts to infer the richness of the BATNA on which their counterpart could rely in case of non-agreement and this type of assessment could deeply affect, among many other aspects, also the responders' fairness considerations on which their decision whether rejecting or not is so heavily based on.

However, from another perspective, as anticipated above, information about the proposer's BATNA could be irrelevant in the UG. In fact, according to the EASI model (emotions as social information, Van Kleef, 2009), there are two paths through which the counterpart's emotional expressions may affect the decision-maker: the strategic and the effective path. Through the strategic path, the others' emotional expressions could be used to infer information about their intentions and positions (for example, their aspirations, their priorities, their BATNA). Through the effective path, the counterpart's emotions influence 
the decision-maker in an immediate and thoughtless way, affecting, for example, interpersonal liking and eliciting affective reactions based on social contagion.

As the responder in a UG has only the power to reject the ultimate proposal and has no possibility to modulate a counteroffer to meet the needs of the counterpart, the responder should be totally de-motivated to follow the strategic path to infer any kind of strategic information about the counterpart. From this functional perspective, we should, therefore, expect the proposers' emotions to affect the responders' rejection rate mainly through the affective path, i.e. influencing the responder's mood and benevolence toward the proposer. Specifically, Harle and Sanfey (2007) found that the rejection rate is more affected by the responders' negative emotions than by the positive ones. In other words, anger increases the rejection tendency while happiness does not significantly reduce it compared to the control group.

As using the proposers' emotional expression to infer their BATNA appears to be strategically futile for the responder according to the EASI model (Van Kleef, 2009), we started our third hypothesis as follows:

H3. Participants would not be motivated to use the proposers' emotions to try to infer their BATNA value. Therefore, the proposers' emotions should not affect the participants' evaluations concerning the richness of the proposers' BATNA.

In summary, our study challenges the assumption that the proposer's emotion cannot convey strategic information to the responder, by introducing the proposer's BATNA as a strategic piece of information that may (or may not) be inferred through his/her emotion. This is intended to fill the gap between the well-established literature on the interpersonal and strategic, effects of the responder's emotion on the proposer and the growing body of literature on the interpersonal effects of proposer's emotion on the responder, which has often neglected to investigate strategic aspects.

\section{Experiment 1}

In Experiment 1, we tested the three hypotheses stated above.

\subsection{Participants and experimental design}

A total of 48 undergraduate students ( 24 women; mean age $=22.68$; SD $=1.87$ ) at the University of Padua (Italy) participated in the study for course credit and the chance to win a prize of 100 euros (which roughly corresponded to an entire month of university tuition fees and in the previous experiment resulted to be perceived as a motivating prize; Pietroni et al., 2009). The one-factor experimental design included the proposer's emotion (anger vs happiness vs no emotion) as a between-participants variable and the decision whether to reject or not the unfair proposer's allocation proposal (offer rejection) and the estimate of the proposer's BATNA as dependent variables. Additional dependent variables were selfreported emotions, the impression of the proposer and manipulation checks.

Participants were randomly assigned to the experimental conditions and the experimenters were blind to this assignment.

All procedures performed in our experiments were in accordance with the ethical standards of the 1964 Helsinki declaration and its later amendments or comparable ethical standards. Informed consent was obtained from all individual participants included in the study.

\subsection{Procedure}

On arrival at the laboratory, participants were welcomed and informed that they would participate in a study on strategic decision-making and that they would be paired with 
IJCMA

33,1

6

another participant who was called "the counterpart" and was seated in a second laboratory (whose behavior was in fact simulated).

Participants then received an explanation of the UG task. All participants learned that they were assigned the role of responder while their counterpart was assigned the role of the proposer. Their task consisted in deciding whether to reject or accept their counterpart's allocation proposal concerning an endowment of 100 chips. If they accepted the offer, the chips would be divided accordingly, if they rejected the offer, both the responder and the proposer would only receive their respective consolation pocket of the chip.

Participants were told that the number of chips included in their consolation pocket depended on the result of the drawing and it could range from 0 to 100 chips. Participants were then invited to draw from an urn a ticket indicating the number of consolation chips they would receive only in case they rejected the counterpart's offer, while they were told that their counterpart was conducting a similar draw in the other laboratory. Actually, the draw was faked and all participants were assigned a consolation pocket of 10 chips, i.e. quite a poor BATNA (the experimental procedure is reported in Figure 1).

To enhance participants' involvement in the task, they were informed that the chips would be converted to lottery tickets at the end of the experiment and that the more points earned, the more lottery tickets one would obtain and the greater would be one's chance of winning a prize of 100 euros.

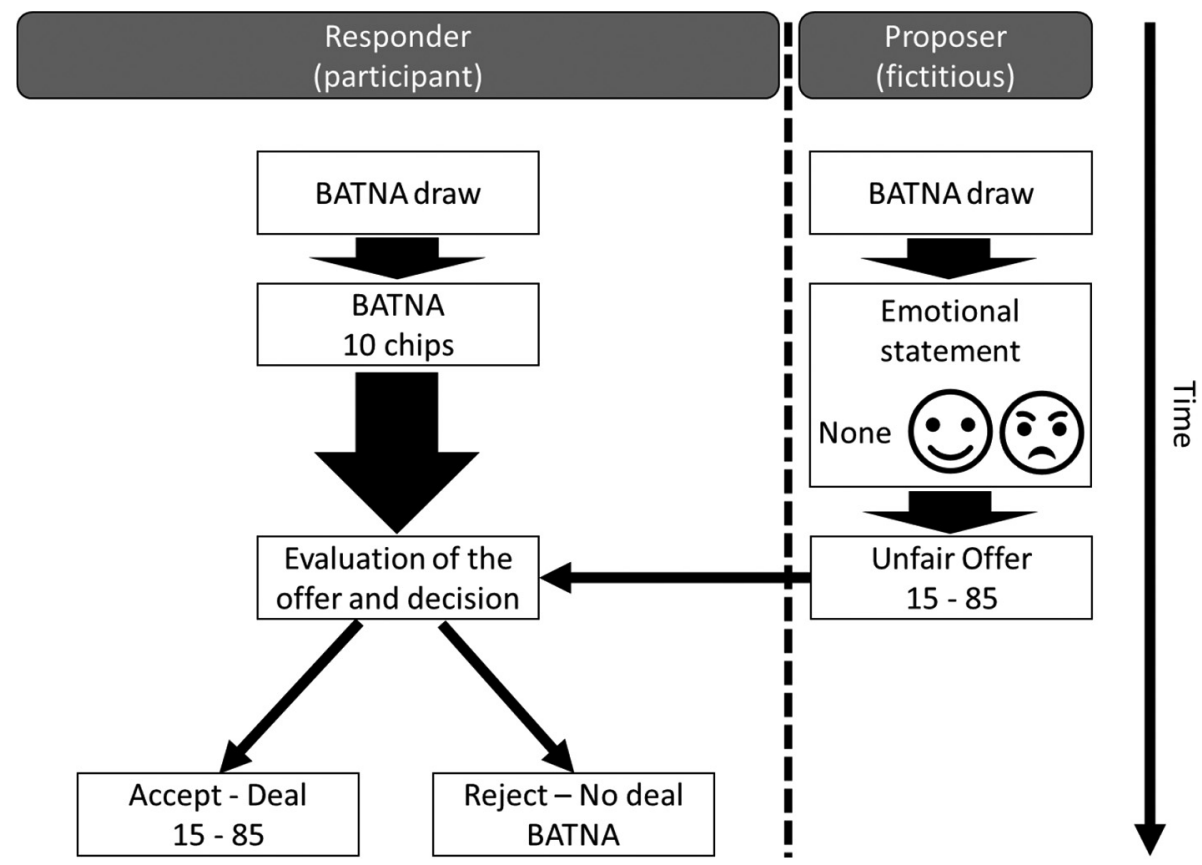

Figure 1. Experimental procedure
Notes: In Experiment 1, the proposer's BATNA was always unknown. In Experiment 2, the proposer's BATNA was either Poor (20 chips), Rich (60 chips) or unknown. The responder's BATNA was always 10 chips 
Just before receiving the proposer's offer, participants learned that an additional goal of the study was to examine the effects of having versus not having information about the counterpart's intentions. They were informed that they were randomly assigned to the "information condition," and therefore that they could read a handwritten note by the proposer with his/her proposal intention, written just after the draw not knowing that it would be read by the participant. A comprehension check and a debriefing verified that the instructions and the rewarding system were understood before proceeding.

After a pause of a couple of minutes, participants received a handwritten note with their counterpart's offer intention (the calligraphy was pre-tested to appear both masculine and feminine). After another minute, participants received a form containing the proposer's actual offer, which indeed corresponded to the intended one: 85 chips for the allocator and 15 chips for the responder/participant. Participants were asked to fill this form marking with a cross either the acceptance option or the rejection option and then to send the form back to the proposer.

Subsequently, we asked participants to take part in some manipulation checks and to assess the emotions they ascribed to the proposer, as well as to themselves. Furthermore, we asked the participants to estimate the proposer's BATNA (i.e. the number of chips drawn as consolation), to express their impression about the proposer and their desire for future interactions with him/her.

At the end of the experiment, participants were thoroughly debriefed and given 3 lottery tickets.

3.2.1 Manipulation of proposer's emotion. The handwritten message with the proposer's offer intention ("I think I will offer 85 chips for me and 15 chips for the other") also contained, except for the control condition, an emotional statement, which constituted the experimental manipulation. It was stressed that the proposer did not know that his or her intentions were going to be revealed to the participant. This was done to lead the participants to believe that they were receiving information about the real emotions of the proposer and not emotions altered for self-presentational or strategic purposes (and in this way also increasing the credibility of the emotion expressed in the written message; Van Leeuwen, 2018). The emotional statements were adapted from Van Kleef et al. (2004a). In the angry proposer condition, the offer intention was preceded by the statement "I am angry for how it is going so far," in the happy proposer condition the offer intention was preceded by the statement "I am happy with how it is going so far."

The verbal emotional expressions have been widely demonstrated to be as effective in conveying the underlying cognitive, affective and motivational states of an individual as non-verbal expressions such as facial and vocal expressions or bodily posture (Van Kleef, 2017; Van Kleef, 2021; Scarantino, 2019).

3.2.2 Dependent measures. The "offer rejection" was the participant's decision to reject the severely unfair proposer's offer consisting of 85 chips for him/herself and only 15 chips for the participant.

To check the adequacy of the manipulation of the proposer's emotion, participants were asked to indicate on a seven-point scale how angry, irritated, happy and satisfied they thought their counterpart was. The items designed to measure perceived anger and irritation correlated substantially $(r=0.87)$ and were averaged into an index of perceived anger. The items pertaining to happiness were combined into an index of perceived happiness $(r=0.77)$.

Participants' own emotions were measured by asking to indicate on a seven-point scale how angry, irritated, happy and satisfied they felt during the task. The items designed to measure negative emotions were averaged into an index of experienced anger $(r=0.74)$. 
IJCMA 33,1

The items pertaining to positive emotion were combined into an index of experienced happiness $(r=0.75)$.

Participants' estimate of the proposer's BATNA was measured by asking them to guess the number of consolation chips they thought their counterpart drew out, i.e. the chips their counterpart received in case of rejection.

Participants' own emotions were measured by asking to indicate on a seven-point scale how angry, irritated, happy and satisfied they felt during the task. The items designed to measure negative emotions were averaged into an index of experienced anger $(r=0.74)$. The items pertaining to positive emotion were combined into an index of experienced happiness $(r=0.75)$.

Impression of the proposer was measured by five differential semantic items. On sevenpoint scale participants assessed how likable, cooperative, disagreeable, cold and bad the counterpart was. Negatively framed items were reverse-scored and the items were combined into a positive impression scale $(\alpha=0.83)$. Finally, participants were asked to express on a seven-point scale their desire to be involved in future interactions with the same proposer.

\subsection{Results}

3.3.1 Manipulation checks. To check the manipulation of the proposer's emotion, we submitted the participants' rating of their opponent's anger and happiness to a 3 (proposer's emotion: anger vs happiness vs no emotion) $\times 2$ (rated emotion: perceived anger vs perceived happiness) ANOVA, the latter variable being a within-participants factor. The hypotheses about the manipulation efficacy were stated as follows. Participants' evaluation of proposer's anger would be higher for participants in anger condition compared to those in the happiness condition; participants' evaluation of proposer's happiness would be higher for participants in happiness condition compared to those in the anger condition; participants' evaluation of proposer's anger and happiness would be equal in the no emotion condition. Results showed the expected interaction between the proposer's emotion and the participants' perception of the proposer's emotion, $F(2,48)=26.2, p<0.001\left(\eta^{2}=0.54\right)$. Participants in the angry proposer condition rated their opponent as significantly angrier $(M=5.03, S D=2.03)$ than did participants in the happy proposer condition $(M=1.25, S D=$ 0.48). Similarly, participants with a happy proposer rated their opponent as happier $(M=6$, $S D=0.97)$ than did participants with an angry one $(M=2.47, S D=1.7)$. Finally, participants who did not read any emotional statements (control condition) rated the proposer not differentially neither angry $(M=3.44 S D=1.86)$ nor happy $(M=3.87, S D=$ 1.85). Further, paired-sample $t$-tests revealed that rating within the two emotion conditions was higher for the intended emotion than for the other emotion: Participants in the angry proposer condition rated their opponent as angrier than happy, $t(15)=2.99, p<0.01$ and those in the happy proposer condition rated their opponent as happier than angry, $t(15)=$ $14.71, p<0.001$. Together, these results indicate that the manipulation of the proposer's emotion was successful.

3.3.2 Offer rejection. To test the effect of the proposer's emotion on the participant's tendency to reject the unfair offer received, we calculated the Chi-square on a 2 (participant's choice: reject vs accept) $\times 3$ (proposer's emotion: anger vs happiness vs no emotion) Contingency Table. The results showed that when the unfair proposal came from a happy proposer it was rejected in a significantly lower number of cases ( 2 out of $16,12.5 \%$ ) than when it came from an angry (10 out of $16,62.5 \%$ ) or a non-emotional (7 out of $16,43.7 \%$ ) counterpart, $\chi^{2}(2)=8.54, p<0.05$. A closer analysis showed that the offer rejection in the happy condition was significantly lower than in the control condition, $\chi^{2}(1)=3.86, p<0.05$, Cramer's $\mathrm{V}=0.42$ and the offer rejection in the control condition was not significantly lower 
than in the angry condition, $\chi^{2}(1)=1.3, n s$ (results are reported in Figure 2). A post hoc power analysis on the Chi-square test was conducted by using the $G^{*}$ Power software (version 3.1.9.4), with $\alpha$ at 0.05. The estimated power $(1-\beta)$ was greater than 0.90 .

3.3.3 Appraisal of the proposer's Batna. A one-factor (proposer's emotion: anger vs happiness vs no emotion) ANOVA on participants' estimate of the proposer's BATNA was performed to test whether the proposer's anger condition would be associated with a poorer estimated BATNA compared to the proposer's happiness and the no emotion conditions. The analysis revealed a significant effect, $F(2,47)=48.36, p<0.001\left(\eta^{2}=0.68\right)$. Participants in the angry proposer condition estimated the proposer's BATNA to be poorer $(M=16.87, S D=9.64)$ than participants in the happy proposer $(M=65.67, S D=17.11)$ and no emotion condition $(M=50.62, S D=15.26)$. An independent-sample $t$-test showed that the appraised BATNA significantly differed between the happy condition and the no emotion condition, $t(30)=2.62, p<0.05$ and the estimated BATNA in the control condition significantly differed from the angry condition, $t(30)=7.48, p<0.001$ (results are reported in Figure 3).

3.3.4 Participants' emotions. To check the participant's experienced emotions, we submitted the participants' rating of their experienced anger and happiness to a 3 (proposer's emotion: anger vs happiness vs no emotion) $\times 2$ (rated emotion: perceived anger vs perceived happiness) ANOVA, the latter variable being a within-participants factor. The hypotheses were stated as follows. Participants' evaluation of experienced anger would be higher for participants in the anger condition compared to those in the happiness and no emotion conditions; participants' evaluation of experienced happiness would be higher for

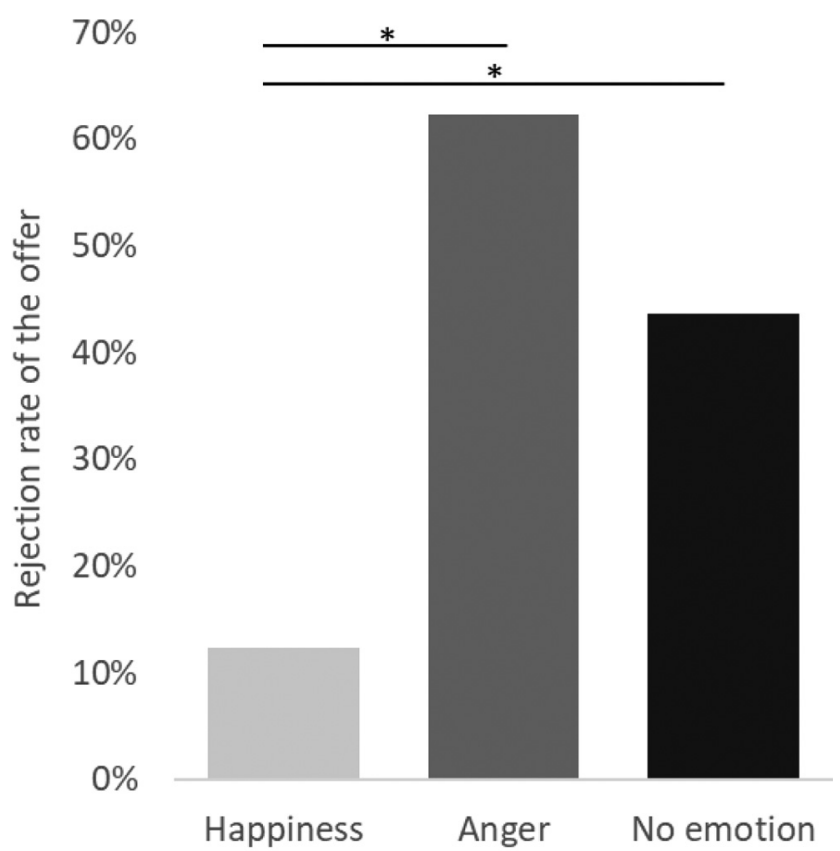

Note: Asterisks indicate significant differences between conditions
Severely unfair ultimatum proposal

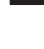


IJCMA

33,1

10

Figure 3.

Mean estimated proposer's BATNA as a function of proposer's emotion in Experiment 1

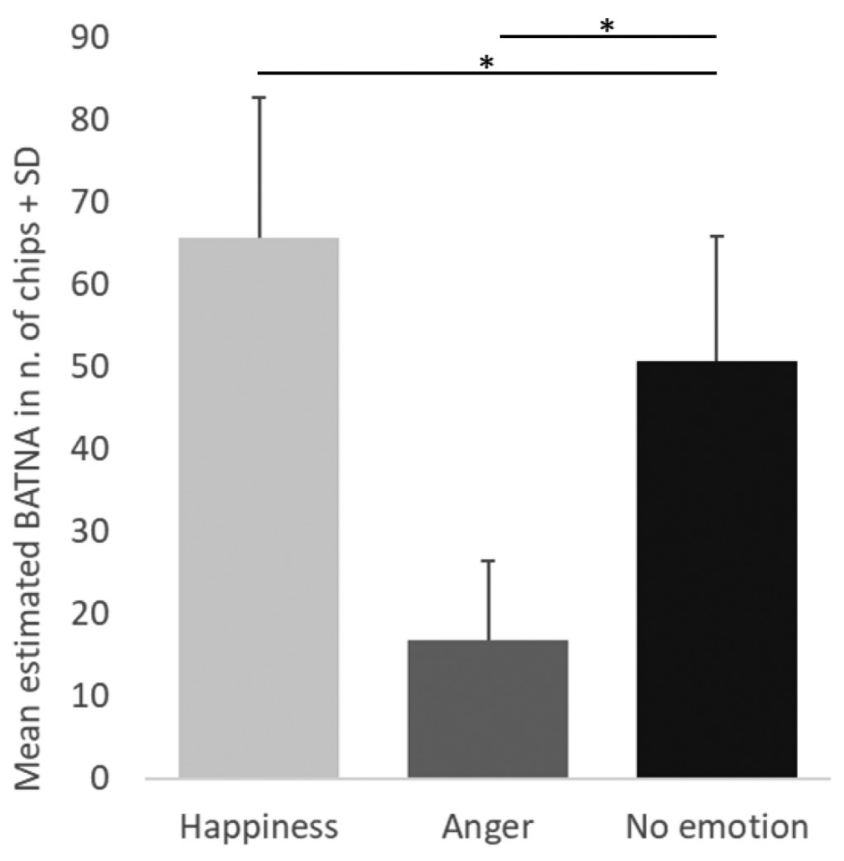

Note: Asterisks indicate significant differences between conditions

participants in the happiness condition compared to those in the anger and no emotion conditions. The ANOVA showed that participants' own emotions were affected by the proposer's emotion. Participants who interacted with an angry proposer reported more experienced anger $(M=2.66, S D=1.3)$ than did participants who interacted with a happy $(M=1.66, S D=0.85)$ or no-emotion $(M=1.91, S D=0.9)$ opponent, $F(2,47)=4.03, p<0.05$ $\left(\eta^{2}=0.15\right)$. Similarly, participants who received the offer from an angry proposer reported less experienced happiness $(M=2.56, S D=1.36)$ than did participants who received the offer from a happy $(M=3.94, S D=1.05)$ or non-emotional $(M=3.66, S D=1.16)$ opponent, $F(2,47)=5.87, p<0.01\left(\eta^{2}=0.21\right)$. This data suggests that, in spite of the minimal interaction with the counterpart, emotional contagion occurred.

3.3.5 Impression of the proposer and desire for future interactions. A one-factor (proposer's emotion: anger vs happiness vs no emotion) ANOVA on the positive impression scale was performed to test whether participants developed a more positive impression of the counterpart in the happiness condition compared to the other conditions. The analysis revealed that the proposer's emotion influenced the participants' impression of the counterpart, $F(2,47)=12.09, p<0.001\left(\eta^{2}=0.35\right)$. Participants developed more positive impressions of happy proposers $(M=4.02, S D=0.61)$ than of non-emotional $(M=2.97$, $S D=0.75)$ and angry $(M=2.8, S D=0.9)$ opponents.

Finally, a one-factor (proposer's emotion: anger vs happiness vs no emotion) ANOVA on the desire to be involved in future interactions was performed, under the hypothesis that participants would express a stronger desire to engage in future interaction in the happiness condition compared to the other conditions. As expected, results show that participants 
expressed a stronger desire to engage in future interactions with happy proposers $(M=4.56$, Severely unfair $S D=1.41)$ rather than non-emotional $(M=3.37, S D=1.41)$ or angry $(M=2.87, S D=1.54)$ opponents, $F(2,47)=5.67, p<0.01\left(\eta^{2}=0.20\right)$.

\subsection{Discussion}

Despite the fact that proposers' emotional expressions consistently influenced the affective state and the social evaluations of the participants, both $H 1$ and $H 2$ cannot be confirmed. In fact, the proposer's anger expression made the participants more irritated and angrier, worsened their impression of the proposer and reduced their desire for future interactions (with moderate to large effects), but despite that, it did not significantly increase the rejection rate compared to the control condition, with a large effect. On the other hand, the proposer's happiness affected both the socio-emotional measures and the behavioral one by reducing the offer rejection tendency compared to the control group. Furthermore, in addition to being effectively and behaviorally influenced by the proposer's emotional expressions, participants demonstrated that they used the other's emotion as a signal to infer the proposers' BATNA (a large effect) in spite of the strategic futility of such information, in disagreement with $\mathrm{H3}$.

As the research illustrates, as rejection decisions on the UG are often based on affective states and fairness-related reasons which are far from the rational prescriptions of the normative economic approach (van't Wout et al., 2006), we could not exclude that also the proposers' BATNA value, inferred through the proposers' emotional expressions, could have affected the participants' rejection rate of the severely unfair proposal. In fact, a higher proposer's BATNA value results in a lower punishment impact of the responder's rejection on the proposer's payoff (Bolton and Zwick, 1995) and maybe also in a tendency to justify and condone the aggressiveness of the severely unfair proposal (Geniole et al., 2017).

To carry out a preliminary check on the plausibility of this hypothesis, we conducted further analysis of Exp 1's data splitting the participants into two statistical groups, the rejecters and the acceptors and checking if their assessment of their proposers' BATNA value was significantly different. An ANOVA with the rejection choice as a factor (reject vs accept) on the appraisal of the proposer's BATNA demonstrated a significant effect, $F(1,47)=9.84$, $p<0.01\left(\eta^{2}=0.17\right)$ indicating that the 19 participants who rejected the offer perceived the proposer's BATNA poorer $(M=31.58, S D=23.87)$ than the 29 participants who accepted the offer $(M=52.76, S D=22.22)$. This further result lends support for the possibility that emotionally inferred BATNA value may "irrationally" contribute to affect the responder's acceptance/rejection choice of a severely unfair proposal.

\section{Experiment 2}

We conducted a second experiment trying to identify which specific path could relate the proposer's happiness with a reduction of the rejection tendency. In fact, the interpersonal effect of the proposer's emotion observed in Experiment 1 on the mitigation of the "rejection bias" could be attributed to three processes:

- the socio-emotive effects of a positive emotional contagion through the affective path;

- the inference of the proposer's BATNA value through a strategic path; or

- an interactive combination of both paths where affective and strategic paths converge.

To check the aforementioned hypotheses and to disentangle the effects of the effective path from the effects of the strategic path, we provided the participants with explicit information concerning the value of the proposers' BATNA. Therefore, in some conditions of this 
IJCMA 33,1

experiment, emotional expressions totally lost their signaling value enabling us to test solely the influence of the effective path. Therefore, we state our hypotheses as follows:

H4. Participants would be always influenced by the proposer's emotion, regardless of the presence of information concerning the proposer's BATNA.

H5. Participants would be not influenced by the proposer's emotion when information regarding BATNA is provided.

H6. Participants would be influenced by the proposer's emotion in interaction with BATNAs' rich and poor conditions.

$H 4$ would lend support to the idea that the proposer's emotion is processed through an affective path, whereas $H 5$ would suggest that the processing follows a strategic path and finally $\mathrm{H} 6$ would indicate that the affective and strategic paths may operate joint influence.

\subsection{Participants and experimental design}

A total of 127 undergraduate students (92 women; mean age $=21.18$; $\mathrm{SD}=1.59$ ) at the University of Padua (Italy) participated in the study for course credit and the chance to win a prize of 100 euros. The sample size was decided after an a priori power analysis with the $\mathrm{G}^{*}$ Power software, using the estimated effect size from Experiment $1(w=0.51)$ and the desired power of 0.90 for a design with six groups, suggested a total sample size of 120 .

The $2 \times 3$ factorial design included the proposer's emotion (anger vs happiness) and proposer's BATNA (poor vs rich vs unknown) as between-participants variables and the decision whether to reject the proposer's unfair allocation proposal (offer rejection) as the dependent variable.

Additional dependent variables were self-reported participant's emotions, the impression of the proposer, desire for future interactions and manipulation checks. Participants were randomly assigned to the experimental conditions and the experimenters were blind to this assignment.

\subsection{Procedure}

The procedure was essentially the same as in Experiment 1. To manipulate the information about the proposer's BATNA in the poor and rich BATNA conditions, when participants were informed that they were assigned to the "information condition" they learned that, besides the opportunity to read their counterpart's intention, they had the possibility to know the number of consolation chips their counterparts received from the draw (the experimental procedure is reported in Figure 1).

In the poor BATNA condition participants were informed that their counterpart drew out 20 chips, in the rich BATNA condition they learned that their counterpart drew out 60 . These values were also defined using as reference the BATNA values inferred by the participants in the angry condition and in the happy condition of Experiment 1.

A comprehension check and a debriefing verified that the instructions and the rewarding system were understood before proceeding.

The manipulation of the proposer's emotion was the same as in Experiment 1.

4.2.1 Dependent measures. The offer rejection was the participants' decision whether to reject or accept the proposer's unfair offer.

To check the adequacy of the manipulation of the proposer's emotion, participants were asked to indicate on a seven-point scale how angry, irritated, happy and satisfied they 
thought their counterpart was. The items designed to measure perceived anger and irritation correlated substantially $(r=0.81)$ and were averaged into an index of perceived anger. The items pertaining to happiness were combined into an index of perceived happiness $(r=0.90)$.

Impression of the proposer was measured by a five items differential semantic scale. On a seven-point scale, participants assessed how likable, cooperative, disagreeable, cold and bad the counterpart was. Negatively framed items were reverse-scored and the items were combined into a positive impression scale $(\alpha=0.72)$.

Finally, participants were asked to express on a seven-point scale their desire to be involved in future interactions with the same proposer.

\subsection{Results}

4.3.1 Manipulation checks. To check the manipulation of the proposer's emotion, we submitted the participants' rating of their opponent's anger and happiness to a 2 (proposer's emotion: anger vs happiness) $\times 3$ (proposer's BATNA: poor vs rich vs unknown) $\times 2$ (rated emotion: perceived anger vs perceived happiness) ANOVA, the latter variable being a withinparticipants factor. The hypotheses about the manipulation efficacy were stated as follows. Participants' evaluation of experienced anger would be higher for participants in the anger condition compared to those in the happiness condition; participants' evaluation of experienced happiness would be higher for participants in the happiness condition compared to those in the anger condition. No effects of BATNA were expected. Results showed the expected two-way interaction between the proposer's emotion and the participants' perception of the proposer's emotion, $F(1,122)=416.93, p<0.001\left(\eta^{2}=0.77\right)$. Participants in the angry proposer condition rated their opponent as significantly angrier $(M=5.49, S D=1.26)$ than did participants in the happy proposer condition $(M=1.94, S D=$ 1.34). Similarly, participants with a happy proposer rated the opponent as happier $(M=5.86$, $S D=1.14)$ than did participants with an angry opponent $(M=2.01, S D=1.09)$. Further, paired-sample $t$-tests revealed that rating within the two emotion conditions was higher for the intended emotion than for the other emotion: Participants in the angry proposer condition rated the opponent as angrier than happy, $t(62)=14.70, p<0.001$ and those in the happy proposer condition rated the opponent as happier than angry, $t(63)=14.49, p<0.001$. There were no effects of BATNA manipulation. Together, these results indicate that the manipulation of the proposer's emotion was successful.

4.3.2 Offer rejection. We first tested the effect of the proposer's emotion on the participant's decision to accept the unfair offer received, calculating the Chi-square on a 2 (participant's choice: reject vs accept) $\times 2$ (proposer's emotion: anger vs happiness) Contingency Table. The results showed that when the unfair proposal came from an angry proposer it was rejected in a significantly higher number of cases $(49,2 \%: 31$ out of 63$)$ than when it came from a happy proposer (25\%: 16 out of 64$), \chi^{2}(2)=7.98, p<0.01$, Cramer's V $=0.25$.

To check whether this effect could be reduced by the information about the proposer's BATNA, we calculated the Chi-square on the same Contingency Table but considering only the participants who received information about the proposer's BATNA, i.e. we excluded from the analysis the "unknown BATNA condition." The results revealed a no more significant Chi-square, $\chi^{2}(1)=1.05, n s$. When participants were aware of the proposer's BATNA value, happy proposers and angry proposers did not obtain a significantly different probability to have their unfair proposal rejected (respectively, 30\%, 12 out of 40 and $41 \%, 16$ out of 39 ).

\section{Severely unfair ultimatum proposal}


IJCMA

33,1

Table 1.

Rejection rate as a function of proposer's emotion and communicated proposer's Batna
Therefore, we proceeded to check the effect of the information about the proposer's BATNA on the participant's tendency to reject the offer. We calculated the Chi-square on a 2 (participant's choice: reject vs accept) $\times 3$ (proposer's BATNA: poor vs rich vs unknown) Contingency Table. The results showed that when the unfair proposal came from a proposer with a poor BATNA, it was rejected in a significantly higher number of cases $(55 \%, 22$ out of 40$)$ than when it came from a proposer with a rich BATNA $(15.4 \%, 6$ out of 39$)$ or unknown BATNA $(39.6 \%, 19$ out of 48$), \chi^{2}(2)=13.51, p<0.01$, Cramer's $\mathrm{V}=0.33$.

Finally, we calculated the specific comparisons between happy and angry conditions within the three BATNA conditions. As is observable in Table 1 and Figure 4, the effect of emotion on rejection emerged only when the proposer's BATNA was not communicated.

4.3.3 Impression of the proposer and desire for future interactions. A 2 (proposer's emotion: anger vs happiness) $\times 3$ (proposer's BATNA: poor vs rich vs unknown) ANOVA on the positive impression scale was performed to test:

- whether participants developed a more positive impression of the counterpart in the happiness condition compared to the anger condition; and

- whether participants developed a more negative impression of the proposers having a poor BATNA compared to the other BATNA conditions.

\begin{tabular}{llcc}
\hline Communicated BATNA & Anger & $\begin{array}{l}\text { Opponent' emotion } \\
\text { Happiness }\end{array}$ & Chi-square \\
\hline Poor BATNA (20 chips) & 11 out of $20(55 \%)$ & 11 out of $20(55 \%)$ & $\chi^{2}(1)=0, n s$ \\
Rich BATNA (60 chips) & 5 out of $19(26.3 \%)$ & 1 out of $20(5 \%)$ & $\chi^{2}(1)=3.4, n s$ \\
Unknown BATNA & 15 out of $24(62.5 \%)$ & 4 out of $24(16.7 \%)$ & $\chi^{2}(1)=10.54$ \\
& & & $p<0.01$, \\
& & Cramer's V $=0.47$ \\
\hline
\end{tabular}

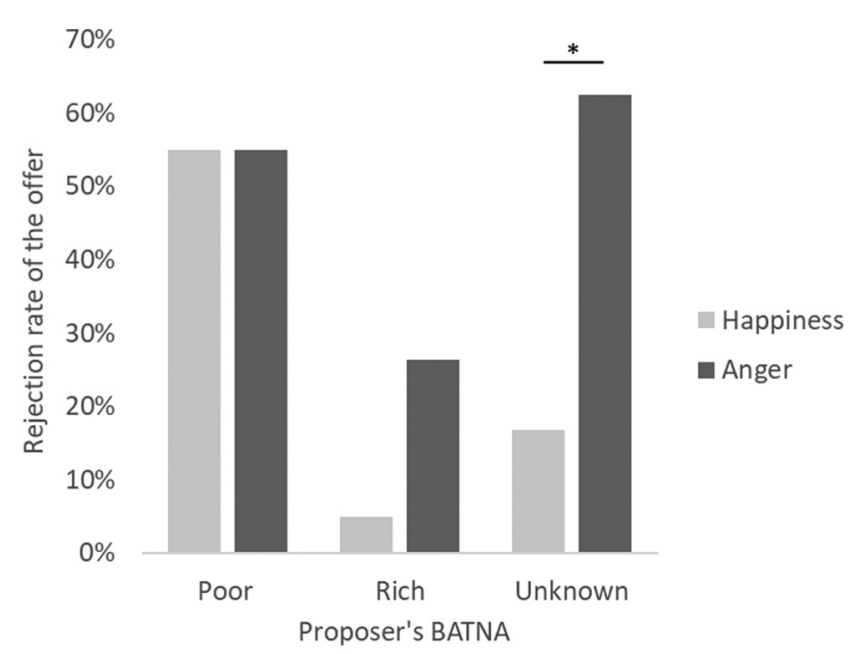

Note: Asterisk indicates a significant difference between conditions
Figure 4.

Rejection rate as a function of proposer's emotion and communicated proposer's BATNA in Experiment 2 
The analysis revealed that the participants' impression of the proposer was affected both by the counterpart's emotion, $F(1,128)=12.25, p<0.001\left(\eta^{2}=0.11\right)$ and by the counterpart's BATNA, $F(2,128)=4.65, p<0.01\left(\eta^{2}=0.07\right)$. Participants developed more positive impressions of happy proposers $(M=3.86, S D=0.81)$ than of angry proposers $(M=3.31$, $S D=0.82)$. Similarly, proposers with a poor BATNA gave a worse impression $(M=3.27$, $S D=0.84)$ than proposers with a rich BATNA $(M=3.7, S D=0.79)$ or unknown BATNA $(M=3.76, S D=0.86)$.

Finally, a 2 (proposer's emotion: anger vs happiness) 3 (proposer's BATNA: poor vs rich vs unknown) ANOVA on the desire to be involved in future interactions was performed under the hypothesis that participants would express a stronger desire to engage in future interaction in the happiness condition compared to the other conditions. Furthermore, ANOVA on the desire for future interactions showed a main effect of proposer's emotion, $F(1,128)=5.17, p<0.05\left(\eta^{2}=0.04\right)$, indicating that participants expressed a stronger desire for future negotiations with happy proposers $(M=4.19, S D=1.67)$ rather than with angry ones $(M=3.51, S D=1.86)$. This effect was qualified with an interaction with the proposer's BATNA, $F(2,128)=3.41, p<0.05\left(\eta^{2}=0.05\right)$, revealing substantially that the strongest desire for future interaction was developed when the happy proposer also had a rich BATNA, i.e. when the opponents appeared positive congruently with the generosity of their BATNA (happy rich BATNA: $M=5.15$; $S D=1.46$; happy poor BATNA: $M=3.71$; $S D=1.49$; happy unknown BATNA: $M=3.79 ; S D=1.69$; angry rich BATNA: $M=3.3 ; S D=$ 1.98; angry poor BATNA: $M=3.7 ; S D=1.45$; angry unknown BATNA: $M=3.54 ; S D=2.13$ ).

\subsection{Discussion}

Once again, the proposer's emotional expression consistently influenced the participant's affective state, the participant's impression of the proposer and the participant's desire to interact again in the future with the same proposer (with moderate effects). Therefore, even in a relationship as short as a UG, we observed emotional contagion and relational implications as systematic interpersonal effects of emotions. However, all these influences were not sufficient to affect the rejection rate of a severely unfair proposal when the proposer's emotion provided no further information in signaling the value of the proposer's BATNA as this information was already explicitly available.

In fact, we found strong support for H5, suggesting that the interpersonal effect of happiness on the strong mitigation of the rejection tendency (as observed in Experiment 1) could be attributed to the "irrational" tendency to formulate the decision whether to reject or accept a severely unfair proposal on the value of the proposers' BATNA. The centrality of this information and the use of the observed emotion to infer it was evident in the "unknown BATNA" condition where the participants, as in Experiment 1, were motivated to compensate for the lack of this information by using emotions as a signal. The consequences were that only in this condition did the proposer's emotional expression affect the rejection rate and, specifically, once again happiness reduced the rejection rate more than anger increased it (with respect to the comparable control group of Exp. 1).

Paradoxically these results demonstrated that in a UG the receiver tends to invest cognitive resources to infer from the proposers' emotion some strategic information and then uses this in an "irrational" way, i.e. to decide whether to reject or accept an extremely unfair proposal on the basis of the strategically irrelevant proposer's own BATNA. In other words, the expresser's emotion is treated strategically to get information that leads to emotionally grounded decisions. In short, the crucial social process in a challenging economic task seems to begin and to conclude emotionally despite the inferential efforts undertaken in the middle of the task.

\section{Severely unfair ultimatum proposal}


IJCMA 33,1
The results also suggest a partial confirmation of $H 6$ limited to a variable, which plays a central role in social relationships and in the economic prosperity fostered by adopting a broader farsighted view (Pietroni and Hughes, 2016): the desire for future interactions. This result supports the suggestive idea that expressing positive emotion cannot be alone an efficient social strategy in the attempt to build loyalty with the other if this display is not congruent with the real availability of satisfying resources. In other words, people do not seem willing to invest in long term relationships with people who appear to bluff or to be inconsistent, expressing happiness despite the fact that they are in an unfortunate position or specifically, happily proposing a poor allocation when they actually have not a generous alternative which could justify such a greedy approach.

\section{General discussion}

Emotions play a crucial role in the UG. Here we join the effort of other scholars proposing a new perspective to understand the effects of emotional expressions in UG, investigating how the proposer's emotional expression influences the responder's affective state, social impression and, above all, the rejection decision to an extremely unfair proposal, i.e. the final and crucial decision which could degenerate a settlement attempt into an open conflict. For the first time, a UG experimental paradigm has been ecologically enhanced by integrating it with a variable and unknown BATNA to be inferred, thus allowing to investigate the strategic role of the interpersonal emotions from the responder's perspective, looking beyond the affective effects investigated in previous experiments. To the sake of completeness, it should be mentioned that BATNA in UG was previously introduced by Van Dijk et al. (2004), but for different purposes and in a different form. In their study, the BATNA was used to attenuate the consequences of rejection to investigate whether the proposer was less concerned with the fairness of the offer, knowing that if the responder had rejected it, they would have received a $10 \%$ reduction of the original offer instead of zero.

\subsection{Theoretical and practical implications}

Our results lend strong support for the interpersonal emotions influence on the responder both through the affective path and through the strategic path, finding also support for a convergent effect of the two on the important responder's desire to build a long-term relationship with the counterpart.

More generally, our study both confirmed the heuristic potential of an emerging experimental approach pioneered by Pietroni et al. (2009) and provided a way to balance past research findings (Van Kleef et al., 2011).

The former refers to the opportunity, in investigating the interpersonal effect of emotions in economic games, to preserve the participants' systemic awareness that all the parts involved in an interaction always have some sort of plan B in case of non-agreement. Following this approach, the parts may be left free to ecologically consider their counterpart's emotional expressions also elicited by her/his BATNA. Actually, emotions are commonly elicited by expectations, therefore the emotions we observe in our counterparts could realistically be triggered by the expectations they are cultivating about alternative scenarios with respect to their contingent relationship with us. Experimental paradigms capable of widening a narrow, local and contingent perspective by providing participants with explicit or implicit BATNAs seem to provide stronger predictive power and external validity.

Regarding the specific effect of the inferred BATNA, we have found that not only it can be inferred through the proposer's emotions but it also seems to influence the perception of fairness or justifiability of a greedy proposal. In fact, the bargaining position of a proposer 
who has a poor BATNA is considered weak, as he/she does not have a good plan B to rely on. In this situation, an unfair proposal is probably perceived as a mere reckless predatory attempt to exploit the responder. On the other hand, a proposer with a richer BATNA could be justified in proposing an aggressive offer as it could be perceived as a fair attempt to moderately further improve her/his already fortunate position. In short, we could accept to be exploited by a powerful happy counterpart but not by a weak, both happy or angry, one. Although these speculations would require further substantiation, we believe they represent interesting perspectives and useful implications on the possible strategic roles of interpersonal emotions expressed by the proposer.

Further, considering the other's BATNA as a possible source of the other's emotions could help balance past research findings. Specifically, research on the EASI model (Van Kleef, 2009) distinguished between converging and competing effects of the affective and strategic path in response to the other's emotions. Convergent effects appear when through both paths, emotions lead the receiver to adopt a collaborative or competitive behavior toward the expresser. Divergent effects appear when one path induces cooperation and the other induces competition as in the most common finding where expressing anger generates negative effects through the affective path (e.g. irritates the receiver) but positive effects through the strategic path (e.g. coveys an image of toughness which yields to more generous concessions).

In the literature, findings of divergent effects are abundant, but research showing convergent effects is still scarce and limited (Pietroni et al., 2009). Investigating happiness effects in BATNA-salient economic games seems a promising way to compensate for this imbalance, as happiness seems remarkably effective in producing both socio-affective and strategic positive convergent effects. In future research, this convergence potential might be tested and expanded into other types of social and economic tasks and in other domains such as persuasion, social influence, social hierarchy, leadership, motivation and team performance (Van Kleef et al., 2011).

In persuasion, for example, a persuader who, in addition to displaying a positive and endearing affective attitude, succeeds in making her/his audience understand that her/ his sunny disposition is motivated by the fact that many others want to take advantage of the limited opportunity she/he is offering, in addition to inducing a social proof effect and positive contagion can create a sense of urgency and scarcity based on the wellknown loss aversion (Cialdini, 2006). In a managerial context, a team leader who, both as a cause or/and as an effect of his positive emotional attitude, is perceived to be able to attract numerous and qualified candidates ready to collaborate with her/him, will induce an implicit and positive spirit of competition that can push each member of the group to give his/her best. On the other side, in the professional contexts in which, unlike in the laboratory, are salient both the systemic and long-term implications of any behavior, it is evident that often, unlike numerous and rigorous experimental findings, those who express anger in negotiation, persuasion, people management, can obtain only momentary and apparent victories.

In all these areas, one might investigate the possibility to solve because of the convergent effects of positive affect, this "expresser strategic dilemma": should I express happiness to appear friendly but with the risk to also appear weak and needy or should I express anger to appear tough but with the risk to undermine the relationship with the counterpart? Finding a scientifically validated way out of this dilemma could, therefore, represent a rigorous and applicative contribution to the promotion of social pacification and socioeconomic growth.

\section{Severely unfair ultimatum proposal}


IJCMA

33,1

18

\section{Conclusion}

Our studies behaviorally corroborate and extend previous findings which have shown that the proposer's positive emotion both modulates the responder's brain activity related to negative feelings prompted by unfair offers (Mussel et al., 2014) and maximizes the acceptance rate compared to negative emotion (Mussel et al., 2013). Importantly, previous studies only investigated the affective path, finding a contagion effect of positive and negative emotions. Here, we take a step further, finding evidence of strategic use of interpersonal emotion which can be used to infer the opponent's BATNA. This particular inferential use of the interpersonal emotions appears to be also ecologically relevant, as the value of each part's own BATNA is carefully kept secret, and therefore these sensitive pieces of information must be indirectly reciprocally estimated exploiting all possible clues.

A potential limitation of the ecological validity of the present study is that participants did not engage in face-to-face interactions. We decided to test our predictions using a paradigm that allowed us to vary the counterpart's emotional expressions as cleanly as possible. Previous research has shown that this task yields data that are similar to data obtained in field settings (Van Kleef et al., 2006) and face-to-face negotiations (Sinaceur and Tiedens. 2006), so we are confident in the generalizability of the findings.

We began this paper by underlining the irrationality, from an economic normative perspective, of rejecting a positive offer in the UG despite the fact that it could be severely unfair. Then we discussed that, in spite of consolidating literature on the interpersonal effects of emotions demonstrating the strategic advantage of expressing anger, research on positive emotions shows that they have an important social function to promote interpersonal relationships and cooperation in a broad range of contexts both personal and organizational (Sels et al., 2021). Attempting to integrate all these perspectives, our study has tried to show that the cold rational prescription of unconditional acceptance could be fulfilled through a warm emotional process, specifically through the exposure to the proposer's happiness in a BATNA salient ecological context.

\section{References}

Arvanitis, A., Papadatou-Pastou, M. and Hantzi, A. (2019), "Agreement in the ultimatum game: an analysis of interpersonal and intergroup context on the basis of the consensualistic approach to negotiation", New Ideas in Psychology, Vol. 54, pp. 15-26, doi: 10.1016/j.newideapsych.2018.12.005.

Bahrya, D.L. and Wilson, R.K. (2006), "Confusion or fairness in the field? Rejections in the ultimatum game under the strategy method", Journal of Economic Behavior and Organization, Vol. 60 No. 1, pp. 37-54, doi: 10.1016/j.jebo.2004.07.005.

Bolton, G.E. and Zwick, R. (1995), "Anonymity versus punishment in ultimatum bargaining”, Games and Economic Behavior, Vol. 10 No. 1, pp. 95-121, doi: 10.1006/game.1995.1026.

Burnham, T. (2007), "High-testosterone men reject low ultimatum game offers", Procedings of the royal Royal Society B, Vol. 274, pp. 2327-2330, doi: 10.1098/rspb.2007.0546.

Cialdini, R.B. (2006), Influence: the Psychology of Persuasion, Revised Edition, William Morrow, New York.

Dunn, B.D., Evans, D., Makarova, D., White, J. and Clark, L. (2012), "Gut feelings and the reaction to perceived inequity: the interplay between bodily responses, regulation, and perception shapes the rejection of unfair offers on the ultimatum game", Cognitive, Affective, and Behavioral Neuroscience, Vol. 12 No. 3, pp. 419-429, doi: 10.3758/s13415-012-0092-z.

Fisher, R., Ury, W. and Patton, B. (2011), Getting to Yes: negotiating Agreement without Giving in, Penguin Books, London.

Geniole, S., MacDonell, E.T. and McCormick, C.M. (2017), "The threat premium in economic bargaining", Evolution and Human Behavior, Vol. 38 No. 5, pp. 572-582, doi: 10.1016/j.evolhumbehav.2016.12.004. 
Gilam, G., Abend, R., Shani, H., Ben-Zion, Z. and Hendler, T. (2019), "The anger-infused ultimatum game: a reliable and valid paradigm to induce and assess anger", Emotion, Vol. 19 No. 1, pp. 84-96, doi: $10.1037 / \mathrm{emo} 0000435$.

Handgraaf, M.J., Van Dijk, E. and De Cremer, D. (2003), "Social utility in ultimatum bargaining”, Social Justice Research, Vol. 16 No. 3, pp. 263-283, doi: 10.1023/A:1025940829543.

Harlé, K.M. and Sanfey, A.G. (2007), "Incidental sadness biases social economic decisions in the ultimatum game”, Emotion, Vol. 7 No. 4, pp. 876-881, doi: 10.1037/1528-3542.7.4.876.

Knez, M.J. and Camerer, C.F. (1995), "Outside options and social comparison in three-player ultimatum

Severely unfair ultimatum proposal game experiments", Games and Economic Behavior, Vol. 10 No. 1, pp. 65-94, doi: 10.1006/ game.1995.1025.

Koenings, M. and Tranel, D. (2007), "Irrational economic decision-making after ventromedial prefrontal damage: evidence from the ultimatum game”, Journal of Neuroscience, Vol. 27 No. 4, pp. 951-956, doi: 10.1523/JNEUROSCI.4606-06.2007.

Lelieveld, G.J., Van Dijk, E., Van Beest, I. and Van Kleef, G.A. (2012), "Why anger and disappointment affect other's bargaining behavior differently: the moderating role of power and the mediating role of reciprocal and complementary emotions", Personality and Social Psychology Bulletin, Vol. 38 No. 9, pp. 1209-1221, doi: 10.1177/0146167212446938.

Liu, C., Chai, J.W. and Yu, R. (2016), "Negative incidental emotions augment fairness sensitivity", Scientific Reports, Vol. 6 No. 1, pp. 1-8, doi: 10.1038/srep24892.

Mussel, P., Göritz, A.S. and Hewig, J. (2013), "The value of a smile: facial expression affects ultimatumgame responses", Judgment and Decision Making, Vol. 8 No. 3, pp. 381-385.

Mussel, P., Hewig, J., Allen, J.J., Coles, M.G. and Miltner, W. (2014), "Smiling faces, sometimes they don't tell the truth: facial expression in the ultimatum game impacts decision making and eventrelated potentials", Psychophysiology, Vol. 51 No. 4, pp. 358-336, doi: 10.1111/psyp.12184.

Nelissen, R.M., Leliveld, M.C., van Dijk, E. and Zeelenberg, M. (2011), "Fear and guilt in proposers: Using emotions to explain offers in ultimatum bargaining", European Journal of Social Psychology, Vol. 41 No. 1, pp. 78-85, doi: 10.1002/ejsp.735.

Ng, G.T.T., Lai, D.C.K., Zeng, X. and Oei, T.P. (2019), “Appreciative joy meditation enhances acceptance of unfair offer in ultimatum game", Mindfulness, Vol. 10 No. 8, pp. 1673-1683, doi: 10.1007/ s12671-019-01139-1.

Park, G. (2021), "The benefit of gratitude: trait gratitude is associated with effective economic decision-making in the ultimatum game", Frontiers in Psychology, Vol. 12, doi: 10.3389/ fpsyg.2021.590132.

Paz, V., Nicolaisen-Sobesky, E., Collado, E., Horta, S., Rey, C., Rivero, M. and Gradin, V.B. (2017), "Effect of self-esteem on social interactions during the ultimatum game", Psychiatry Research, Vol. 252, pp. 247-255, doi: 10.1016/j.psychres.2016.12.063.

Pietroni, D. and Hughes, S.V. (2016), "Nudge to the future: capitalizing on illusory superiority bias to mitigate temporal discounting", Mind and Society, Vol. 15 No. 2, pp. 247-264, doi: 10.1007/ s11299-016-0193-4.

Pietroni, D., Van Kleef, G.A., De Dreu, C.K.W. and Pagliaro, S. (2008), "Emotions as strategic information: effects of other's emotional expressions on fixed-pie perception, demands, and integrative behavior in negotiation", Journal of Experimental Social Psychology, Vol. 44 No. 6, pp. 1444-1454, doi: 10.1016/j.jesp.2008.06.007.

Pietroni, D., Van Kleef, G.A., Rubaltelli, E. and Rumiati, R. (2009), "When happiness pays in negotiation: the interpersonal effects of exit-option directed emotions", Mind and Society, Vol. 8 No. 1, pp. 77-92, doi: 10.1007/s11299-008-0047-9.

Reed, L.I., Okun, S. and Cooley, C. (2020), "The intrapersonal and interpersonal effects of anger in ultimatum bargaining", Adaptive Human Behavior and Physiology, Vol. 6 No. 2, pp. 236-248, doi: 10.1007/s40750-020-00136-2. 
IJCMA 33,1

Scarantino, A. (2019), Affective Pragmatics Extended: From Matural to Overt Expressions of Emotions, In The Social Nature of Emotion Expression, Springer, Cham.

Sels, L., Tran, A., Greenaway, K.H., Verhofstadt, L. and Kalokerinos, E.K. (2021), "The social functions of positive emotions", Current Opinion in Behavioral Sciences, Vol. 39, pp. 41-45, doi: 10.1016/j. cobeha.2020.12.009.

Sinaceur, M. and Tiedens, L.Z. (2006), "Get mad and get more than even: when and why anger expression is effective in negotiations", Journal of Experimental Social Psychology, Vol. 42 No. 3, pp. 314-322, doi: 10.1016/j.jesp.2005.05.002.

Solnick, S.J. and Schweitzer, M.E. (1999), "The influence of physical attractiveness and gender on ultimatum game decisions", Organizational Behavior and Human Decision Processes, Vol. 79, pp. 199-215, doi: 10.1006/obhd.1999.2843.

Van Dijk, E., de Cremer, D. and Handgraaf, M.J.J. (2004), "Social value orientations and the strategic use of fairness in ultimatum bargaining", Journal of Experimental Social Psychology, Vol. 40 No. 6, pp. 697-707, doi: 10.1016/j.jesp.2004.03.002.

Van Dijk, E., Van Kleef, G.A., Steinel, W. and Van Beest, I. (2008), "A social functional approach to emotions in bargaining: when communicating anger pays and when it backfires", Journal of Personality and Social Psychology, Vol. 94 No. 4, pp. 600-614, doi: 10.1037/00223514.94.4.600.

Van Kleef, G.A. (2009), "How emotions regulate social life: the emotions as social information (EASI) model", Current Directions in Psychological Science, Vol. 18 No. 3, pp. 184-188, doi: 10.1111/j.14678721.2009.01633.x.

Van Kleef, G.A. (2017), "The social effects of emotions are functionally equivalent across expressive modalities", Psychological Inquiry, Vol. 28 Nos 2/3, pp. 211-216, doi: 10.1080/1047840X.2017.1338102.

Van Kleef, G.A. (2021), "Comment: moving (further) beyond private experience: on the radicalization of the social approach to emotions and the emancipation of verbal emotional expressions", Emotion Review, Vol. 13 No. 2, pp. 90-94, doi: 10.1177/1754073921991231.

Van Kleef, G.A. and Côté, S. (2018), "Emotional dynamics in conflict and negotiation: individual, dyadic, and group processes", Annual Review of Organizational Psychology and Organizational Behavior, Vol. 5 No. 1, pp. 437-464, doi: 10.1146/annurev-orgpsych-032117-104714.

Van Kleef, G.A., De Dreu, C.K.W. and Manstead, A.S.R. (2004a), "The interpersonal effects of anger and happiness in negotiations", Journal of Personality and Social Psychology, Vol. 86 No. 1, pp. 57-76, doi: 10.1037/0022-3514.86.1.57.

Van Kleef, G.A., De Dreu, C.K.W., Pietroni, D. and Manstead, A.S.R. (2006), "Power and emotion in negotiation: power moderates the interpersonal effects of anger and happiness on concession making”, European Journal of Social Psychology, Vol. 36 No. 4, pp. 557-581, doi: 10.1002/ejsp.320.

Van Kleef, G.A., DeDreu, C.K.W. and Manstead, A.S.R. (2010), "An interpersonal approach to emotion in social decision making: the emotions as social information model”, Advances in Experimental Social Psychology, Vol. 42, pp. 45-96, doi: 10.1016/S0065-2601(10)42002-X.

Van Kleef, G.A., Van Doorn, E.A., Heerdink, M.W. and Koning, L.F. (2011), "Emotion is for influence", European Review of Social Psychology, Vol. 22 No. 1, pp. 114-163, doi: 10.1080/10463283.2011.627192.

Van Leeuwen, B., Noussair, C.N., Offerman, T., Suetens, S., Van Veelen, M. and Van De Ven, J. (2018), "Predictably anger - facial cues provide a credible signal of destructive behavior", Management Science, Vol. 64 No. 7, pp. 3352-3364, doi: 10.2139/ssrn.2535057.

Yamagishi, T., Horitaa, Y., Takagishia, H., Shinadaa, M., Tanidaa, S. and Cookb, K.S. (2009), “The private rejection of unfair offers and emotional commitment", Proceedings of the National Academy of Sciences, Vol. 106 No. 28, pp. 11520-11523, doi: 10.1073/pnas.0900636106.

Zheng, Y., Yang, Z., Jin, C., Qi, Y. and Liu, X. (2017), "The influence of emotion on fairness-related decision making: a critical review of theories and evidence", Frontiers in Psychology, Vol. 8 No. 1592, pp. 1-10, doi: 10.3389/fpsyg.2017.01592. 


\section{Further reading}

Güth, W., Schmittberger, R. and Schwarze, B. (1982), "An experimental analysis of ultimatum bargaining”, Journal of Economic Behavior and Organization, Vol. 3 No. 4, pp. 367-388, doi: 10.1016/0167-2681(82)90011-7.

Nowak, M.A., Page, K.M. and Sigmund, K. (2000), "Fairness versus reason in the ultimatum game", Science, Vol. 289 No. 5485, pp. 1773-1775, doi: 10.1126/science.289.5485.1773.

Sanfey, A.G., Rilling, J.K., Aronson, J.A., Nystrom, L.E. and Cohen, J.D. (2003), "The neural basis of economic decision making in the ultimatum game”, Science, Vol. 300 No. 5626, pp. 1755-1758, doi: 10.1126/science.1082976.

Scarantino, A. (2019), Affective Pragmatics Extended: From Natural to Overt Expressions of Emotions, the Social Nature of Emotion Expression, Springer, Cham.

Sinaceur, M., Van Kleef, G.A., Neale, M.A., Adam, H. and Haag, C. (2011), "Hot or cold: is communicating anger or threats more effective in negotiation?", Journal of Applied Psychology, Vol. 96 No. 5, pp. 1018-1032, doi: 10.1037/a0023896.

Steinel, W., Van Kleef, G.A. and Harinck, F. (2008), “Are you talking to me?! Separating the people from the problem when expressing emotions in negotiation", Journal of Experimental Social Psychology, Vol. 44 No. 2, pp. 362-369, doi: 10.1016/j.jesp.2006.12.002.

Thaler, R.H. (1988), “Anomalies: the ultimatum game”, Journal of Economic Perspectives, Vol. 2 No. 4, pp. 195-206.

Van Kleef, G.A. and De Dreu, C.K.W. (2010), "Longer-term consequences of anger expression in negotiation: retaliation or spill-over?", Journal of Experimental Social Psychology, Vol. 46 No. 5, pp. 753-760, doi: 10.1016/j.jesp.2010.03.013.

Van't Wout, M., Kahn, R.S., Sanfey, A.G. and Aleman, A. (2006), “Affective state and decision-making in the ultimatum game”, Experimental Brain Research, Vol. 169 No. 4, pp. 564-568, doi: 10.1007/ s00221-006-0346-5.

\section{Author affiliations}

Davide Pietroni, Department of Neuroscience, Imaging and Clinical Sciences, University "G. d'Annunzio" of Chieti-Pescara, Pescara, Italy

Sibylla Hughes Verdi, University of Padua, Padua, Italy

Felice Giuliani, Department of Neuroscience, Imaging and Clinical Sciences, University "G. d'Annunzio" of Chieti-Pescara, Pescara, Italy

Angelo Rosa, Department of Management, Finance and Technology, University “LUM”, Casamassima, Italy

Fabio Del Missier, Department of Life Sciences, University of Trieste, Trieste, Italy, and

Riccardo Palumbo, Department of Neuroscience, Imaging and Clinical Sciences, Gabriele d'Annunzio University of Chieti and Pescara, Pescara, Italy and Center for Advanced Studies and Technologies “CAST", University "G. d'Annunzio" of Chieti-Pescara, Pescara, Italy

\section{Corresponding author}

Felice Giuliani can be contacted at: felice.giuliani@unich.it

Severely unfair

ultimatum proposal 